\section{Evolución del riesgo cardiovascular y sus factores en Temuco entre 1989 y $2011-12$}

\author{
FERNANDO LANAS MSC ${ }^{1,2,3}$, PAMELA SERÓN MSC ${ }^{1,2}$, TERESA PUIG ${ }^{4}$
}

\section{Cardiovascular risk factors among males in 1989 and 2011-12 in a southern Chilean city}

Background: A successful cardiovascular prevention program should induce a reduction of risk factors along time. Aim: To assess changes in cardiovascular risk factors among males aged between 35 and 65 years living in Southern Chile. Material and Methods: The results of two cross sectional household surveys, with a probability sampling stratified by socioeconomic status, were analyzed. Two hundred males were evaluated in 1989 and 800 in 2011-12, paired by age for selection. Results: In the second survey, a mean weight increase of $4.5 \mathrm{~kg}$ was recorded. Body mass index increased from 27.1 to $28.6 \mathrm{~kg} / \mathrm{m}^{2}(p<0.01)$, especially in men younger than 45 years old. No changes in smoking prevalence were observed. The prevalence of hypertension and hypertensive patients in treatment increased from 32.7 to $38.1 \%$ and from 17 to 33\%, respectively. The number of treated hypertensive patients with a well-controlled blood pressure did not change significantly. In 1989 and 2011-12, mean total cholesterol values were 192 and $201 \mathrm{mg} / \mathrm{dl}$ respectively $(p<0.01)$. The figures for mean non-HDL cholesterol were 152 and $160 \mathrm{mg} / \mathrm{dl}(p=0.03)$. The frequency of people with total cholesterol over $240 \mathrm{mg} / \mathrm{dl}$ or using statins increased from 15 to $25 \%(p<0.01)$. The estimated 10 years risk of myocardial infarction and coronary death using Framingham tables was 9,0 in both periods ( $p=0.95$ ). Conclusions: In a 22 years period an increase in the prevalence of obesity and elevated total cholesterol was observed. There was a higher proportion of individuals treated for hypertension and dyslipidemia, but without reduction in the estimated cardiovascular risk.

(Rev Med Chile 2014; 142: 467-474)

Key words: Coronary artery disease; Prevalence; Risk factors
'Departamento de Medicina Interna. Facultad de Medicina. Universidad de La Frontera, Temuco, Chile.

${ }^{2}$ Centro de Excelencia CIGES. ${ }^{3}$ Estudiante PhD, Universidad Autónoma de Barcelona, España. ${ }^{4}$ Servicio de Epidemiología Clínica y Salud Pública (IIB-Sant Pau). Hospital Santa Creu i Sant Pau, Barcelona, España. Universidad Autónoma de Barcelona, España.

Financiamiento: INCLEN/ Fundación Rockefeller y National Heart, Lung, and Blood Institute (NHLBI) grant number HHSN268200900029C"

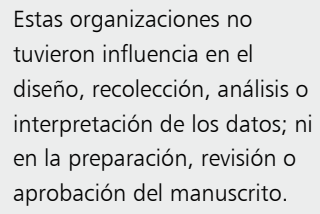

Recibido el 25 de enero de 2014, aceptado el 22 de abril de 2014.

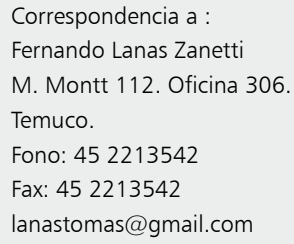

E n Chile, las enfermedades cardiovasculares son la primera causa de muerte y una de las condiciones con carga de enfermedad más elevada, predominando el accidente vascular encefálico (AVE) y el infarto del miocardio (IAM) ${ }^{1-3}$. De acuerdo a los resultados de los estudios INTERHEART e INTERSTROKE los factores de riesgo cardiovasculares determinan cerca de $90 \%$ de ambas condiciones ${ }^{4-6}$ y su tratamiento tiene impacto significativo en el pronóstico de estos pacientes ${ }^{7,8}$.

En los últimos años existen datos nacionales que apoyan la posibilidad de una reducción de la mortalidad cardiovascular ${ }^{9}$ y paradojalmente de aumento en la prevalencia de los factores de riesgo cardiovascular, en especial el sobrepeso y obesidad $^{10-14}$, que se asocian fuertemente a la prevalencia de hipertensión arterial, dislipidemia y diabetes mellitus ${ }^{15,16}$. Por otro lado, la hipertensión arterial y diabetes mellitus fueron incorporadas al Régimen de Garantías Explícitas en Salud, garantizando el Estado a toda la población acceso y financiamiento de la atención, se establecieron guías de práctica clínica, implementándose a partir del año $2005^{17}$, por lo que se podría esperar un mejor 
control de los factores de riesgo en la población.

El propósito de este estudio es evaluar los cambios producidos en la prevalencia de factores de riesgo, y del riesgo cardiovascular global, en la comuna de Temuco, en hombres entre 35 y 65 años entre 1989 y 2011-12.

\section{Diseño}

Comparación entre los resultados de 2 estudios de corte transversal realizados en 1989 y en 2011-12, con igual metodología, en la ciudad de Temuco.

\section{Participantes}

En ambos estudios se realizó un muestreo probabilístico multietápico. En primer lugar se seleccionaron sectores de la ciudad estratificados por nivel socioeconómico, luego se seleccionaron hogares usando muestreo sistemático y finalmente en cada hogar se eligió sólo un sujeto varón en el rango de edad determinado. El estudio del año 1989 incluyó 200 hombres entre 35 y 65 años sin historia de enfermedad coronaria o cerebrovascular $^{18}$, estos se compararon con 800 hombres, pareados por edad con un rango de 1 año máximo de diferencia, sin historia de enfermedad coronaria o cerebrovascular, seleccionados en forma aleatoria del corte transversal basal del estudio CESCAS, realizado los años 2011-12 ${ }^{19}$. Los criterios de selección fue ser residente de la ciudad y aceptarparticipar. La tasa de aceptación a participar fue $77 \%$ en 1989 y $82 \%$ el 2011-12.

\section{Variables}

Se determinó sobrepeso y obesidad, tabaquismo, hipertensión arterial, colesterol total y HDL, y riesgo de IAM o muerte coronaria a 10 años estimado a partir de tablas de Framingham ${ }^{20}$.

Definiciones: se definió obesidad con Índice de Masa Corporal (IMC) $\geq 30 \mathrm{~kg} / \mathrm{m}^{2}$ y sobrepeso con IMC $\geq 25 \mathrm{~kg} / \mathrm{m}^{2}$. Fumador a quien al momento de la entrevista fumaba al menos 1 cigarrillo al día en promedio y ex fumador a quien no ha fumado el último año. Hipertensión arterial con presión sistólica promedio $\geq 140 \mathrm{~mm} \mathrm{Hg}$, presión diastólica promedio $\geq 90 \mathrm{~mm} \mathrm{Hg}$, o tomando fármacos hipotensores $^{21}$. Colesterol elevado: sujetos con colesterol total $\geq 240 \mathrm{mg} / \mathrm{dL}$ o en tratamiento con fármacos hipolipidemiantes. Colesterol HDL reducido $<40 \mathrm{mg} / \mathrm{dL}^{20}$. El IMC se calculó como el cociente entre peso y estatura elevada al cuadrado.
La presión arterial se calculó como el promedio de las 2 mediciones, el colesterol no HDL se calculó como la diferencia entre colesterol total y $\mathrm{HDL}^{20}$. Se calculó el riesgo a 10 años según tablas de Framingham para ambos períodos, utilizando los valores de edad, sexo, tabaquismo, colesterol total y presión sistólica ${ }^{20}$. Para el análisis se subdividió ambos grupos por edad con rangos de 35-44, 45-54 y 55-65 años, y por nivel de educación: $<8$ años, entre 8-12 y > 12 años.

\section{Fuente de datos/mediciones}

En el domicilio se realizó una encuesta que incluía información demográfica, sobre historia médica, factores de riesgo y terapia. Luego, los participantes fueron citados a un centro de salud donde se realizó mediciones de peso, estatura, presión arterial y se tomó una muestra de sangre en ayunas para determinar colesterol total y HDL, cuyos resultados fueron validados por un laboratorio acreditado en Australia en 1989 y Argentina en 2011-12. El peso y estatura se determinó en ropa ligera, y fue registrado con un decimal. La presión arterial se determinó como el promedio de 2 mediciones con esfigmomanómetro de mercurio, siguiendo las recomendaciones de la American Heart Association ${ }^{21}$. En ambos estudios se siguieron los mismos procedimientos.

\section{Tamaño muestra}

Se calculó para una prevalencia de un factor de riesgo de $20 \%$ en el primer estudio y relación de prevalencias de 1,5 entre el primer y segundo estudio. Para un poder de $80 \%$ y valor alfa 0,05 se requiere 198 y 791 sujetos respectivamente ${ }^{22}$.

\section{Análisis estadístico}

Análisis descriptivo: para variables continuas se calculó media y desviación estándar, para variables categóricas, frecuencias absolutas y relativas. Las diferencias en factores de riesgo se analizaron usando chi cuadrado para proporciones y tendencias y test de Student para variables continuas, en caso que el test de Bartlett demuestre diferencias en las varianzas de los grupos se usó la prueba de Kruskal-Wallis para 2 grupos. Para el análisis estadístico se usó el programaStata11@.

Ambos estudios fueron aprobados por el Comité de Ética del Servicio de Salud Araucanía Sur y los pacientes firmaron un consentimiento informado. 
Evolución de los factores de riesgo cardiovascular - F. Lanas et al

\section{Resultados}

La edad media fue similar en ambos grupos. Hubo un aumento de peso promedio de $4,4 \mathrm{~kg}$, con un aumento de estatura promedio de $4 \mathrm{~mm}$, como consecuencia el IMC aumentó significativamente de $27,1 \mathrm{~kg} / \mathrm{m}^{2}$ a $28,6 \mathrm{~kg} / \mathrm{m}^{2}$ (Tabla 1 ). Hubo un aumento significativo de la prevalencia de obesidad y de la suma de sobrepeso y obesidad los años 2011-12, de modo que en 1989 uno de cada 3 hombres tenía peso normal y en 2011-12 menos de uno de cada cinco (Tabla 2). La obesidad mórbida (IMC $>35 \mathrm{~km} / \mathrm{m}^{2}$ ) también aumentó de $3 \%$ a $6,6 \%$. Al dividir los grupos por intervalos de edad se observó el año 1989 un aumento progresivo del IMC desde $26,5 \pm 5,1 \mathrm{~kg} / \mathrm{m}^{2}$ para las edades entre 35-44 años, a 27,0 \pm 4,6 en el rango 45-54 años, llegando a 28,1 $\pm 3,8$ entre 55-65 años

Tabla 1. Características demográficas, clínicas y niveles de colesterol en los años 1989 y 2011-12. Valores promedio y desviación estándar

\begin{tabular}{|lrcc|}
\hline Año & \multicolumn{1}{c}{$\mathbf{1 9 8 9}$} & $\mathbf{2 0 1 1 - 1 2}$ & valor p \\
Edad (años) & $47,8 \pm 9,4$ & $47,7 \pm 8,7$ & 0,89 \\
Estudios (años) * & $9,7 \pm 4,4$ & $12,6 \pm 5,6$ & $<0,0001$ \\
Estatura (cm) & $168,8 \pm 6,3$ & $169,2 \pm 7,3$ & 0,57 \\
Peso $(\mathrm{kg})$ & $77,3 \pm 14,5$ & $81,8 \pm 13,6$ & $<0,0001$ \\
IMC $\left(\mathrm{kg} / \mathrm{m}^{2}\right)$ & $27,1 \pm 4,6$ & $28,6 \pm 4,2$ & $<0,0001$ \\
Presión sistólica $(\mathrm{mmHg})$ & $130,4 \pm 17,5$ & $128,4 \pm 18,9$ & 0,16 \\
Presión diastólica (mmHg) & $85,4 \pm 12,7$ & $84,4 \pm 12,5$ & 0,038 \\
Colesterol total (mg/dL) * & $192,2 \pm 50,1$ & $201,2 \pm 52,2$ & 0,0013 \\
Colesterol HDL (mg/dL) & $40,0 \pm 9,8$ & $41,6 \pm 12,7$ & 0,1 \\
Colesterol no HDL (mg/dL) * & $152,2 \pm 49,8$ & $159,6 \pm 40,9$ & 0,03 \\
Riesgo eventos CV a 10 años (\%) & $9,0 \pm 7,3$ & $90,5 \pm 74,0$ & 0,95 \\
\hline
\end{tabular}

*test de Kruskal-Wallis. IMC: índice de masa corporal.

Tabla 2. Prevalencia de los factores de riesgo en el año 1989 y 2011-12 en Temuco

\begin{tabular}{|c|c|c|c|c|c|}
\hline \multirow{2}{*}{$\begin{array}{l}\text { Año } \\
\text { Sobrepeso }\end{array}$} & \multicolumn{2}{|c|}{$\begin{array}{c}1989 \\
\text { Prevalencia IC 95\% }\end{array}$} & \multicolumn{2}{|c|}{$\begin{array}{c}2011-12 \\
\text { Prevalencia IC 95\% }\end{array}$} & \multirow{2}{*}{$\begin{array}{c}\text { Valor } \mathbf{p} \\
0,15\end{array}$} \\
\hline & 43,0 & $36,0-50,2$ & 51,6 & $48,0-55,1$ & \\
\hline Obesidad & 24,0 & $18,3-30,5$ & 31,1 & $28,4-34,9$ & 0,048 \\
\hline Sobrepeso y obesidad & 67,0 & $60,0-73,5$ & 83,1 & $80,3-85,6$ & $<0,0001$ \\
\hline Fumador & 33,5 & $27,0-40,5$ & 36,9 & $33,5-40,3$ & 0,22 \\
\hline Exfumador & 33,0 & $26,5-40,2$ & 36,9 & $33,5-40,3$ & 0,009 \\
\hline Colesterol total elevado* & 15,0 & $10,1-20,0$ & 25,4 & $22,4-28,4$ & 0,0019 \\
\hline Colesterol HDL < 40 (mg/dL) & 48,5 & $41,4-55,7$ & 51,7 & $44,4-58,6$ & 0,43 \\
\hline Hipertensión arterial (\%) & 32,7 & $26,1-39,5$ & 38,1 & $34,7-41,5$ & 0,14 \\
\hline Conocimiento hipertensión (\%) & 50,0 & $30,1-67,8$ & 53,9 & $48,2-59,6$ & 0,68 \\
\hline Tratamiento hipertensión (\%) & 16,7 & $3,4-30,0$ & 32,9 & $27,7-38,5$ & 0,07 \\
\hline Presión controlada (\%) & 6,7 & $0-15,6$ & 13,8 & $9,5-17,7$ & 0,26 \\
\hline
\end{tabular}

${ }^{*}$ Colesterol total elevado: $\geq 240 \mathrm{mg} / \mathrm{dl}$ o uso de fármacos hipolipidemiantes. IC 95\%: intervalo de confianza de $95 \%$. 
( $\mathrm{p}<0,01)$. En cambio en los años 2011-12 el IMC fue $28,6 \pm 4,3 \mathrm{~kg} / \mathrm{m}^{2}$ entre $35-44$ años y el IMC y la prevalencia de obesidad se mantiene estable en las décadas de edad siguientes (Tabla 3 y 4, Figura 1). El mejor nivel educacional, más de 12 años de estudios, se asoció a una menor prevalencia de obesidad en $1989,16,7 \%$, pero en 2011-12 la prevalencia en esta categoría fue $35,4 \%$ (Tabla 5 ).

La proporción de fumadores aumentó de $33,5 \%$ a $36,9 \%$, pero la diferencia no fue estadísticamente significativa, como tampoco la suma de fumadores y exfumadores. El nivel educacional

Tabla 3. Promedio y desviación estándar de IMC, presión arterial, colesterol total y riesgo cardiovascular a 10 años por rango de edad

\begin{tabular}{|lcccccc|}
\hline Año & $\begin{array}{c}\text { Rango edad } \\
\text { (años) }\end{array}$ & $\begin{array}{c}\text { IMC } \\
\left(\mathbf{k g} / \mathbf{m}^{\mathbf{2}}\right)\end{array}$ & $\begin{array}{c}\text { Presión sistólica } \\
\mathbf{( m m H g )}\end{array}$ & $\begin{array}{c}\text { Presión diastólica } \\
\mathbf{( m m H g )}\end{array}$ & $\begin{array}{c}\text { Colesterol total } \\
\text { (mg/dL) }\end{array}$ & $\begin{array}{c}\text { Riesgo CV } \\
\mathbf{a ~ 1 0} \mathbf{~ a n ̃ o s}\end{array}$ \\
\hline 1989 & $35-44$ & $26,5 \pm 5,1^{*}$ & $124,1 \pm 12,1^{*}$ & $82,5 \pm 8,9^{*}$ & $193,7 \pm 48,3$ & $5,5 \pm 6,0^{*}$ \\
& $45-54$ & $27,0 \pm 4,6$ & $130,3 \pm 18,1$ & $85,6 \pm 9,2$ & $187,7 \pm 44,6$ & $8,2 \pm 6,3$ \\
& $55-65$ & $28,1 \pm 3,8$ & $139,6 \pm 19,5$ & $89,5 \pm 18,6$ & $194,7 \pm 60,5$ & $15,0 \pm 6,4$ \\
$2011-12$ & $35-44$ & $28,6 \pm 4,3$ & $123,9 \pm 15,8^{*}$ & $82,3 \pm 12,2^{*}$ & $201,8 \pm 41,9$ & $4,5 \pm 5,1^{*}$ \\
& $45-54$ & $28,5 \pm 4,0$ & $128,6 \pm 17,3$ & $83,6 \pm 12,0$ & $198,7 \pm 41,2$ & $10,0 \pm 7,0$ \\
& $55-65$ & $28,5 \pm 4,3$ & $135,9 \pm 19,5$ & $84,6 \pm 11,2$ & $202,9 \pm 44,5$ & $15,9 \pm 6,1$ \\
\hline
\end{tabular}

*p para tendencia en el mismo período <0,01. IMC: índice de masa corporal.

Tabla 4. Prevalencia de factores de riesgo por rango de edad e intervalo de confianza de $95 \%$

\begin{tabular}{|c|c|c|c|c|c|c|c|}
\hline \multirow{2}{*}{$\begin{array}{l}\text { Año } \\
1989\end{array}$} & \multirow{2}{*}{$\begin{array}{c}\text { Rango } \\
\text { edad } \\
35-44\end{array}$} & Sobrepeso & Obesidad & Fumador & $\begin{array}{c}\text { Hipertensión } \\
\text { arterial }\end{array}$ & \multicolumn{2}{|c|}{$\begin{array}{l}\text { Colesterol } \\
\text { elevado }\end{array}$} \\
\hline & & $43,4 \quad 32,5-54,7$ & $17,8 * 10,5-28,1$ & $49,4 \quad 38,2-60,6$ & $19,3 * 11,4-29,2$ & 13,3 & $6,0-20,6$ \\
\hline & $45-54$ & $36,7 \quad 24,6-50,1$ & $26,7 \quad 16,1-39,7$ & $26,7 \quad 16,1-39,7$ & $30,0 \quad 18,9-43,2$ & 15,0 & $5,9-24,0$ \\
\hline & $55-65$ & $49,1 \quad 35,6-62,7$ & $29,8 \quad 18,4-43,4$ & $17,5^{*} \quad 8,8-29,9$ & $54,4 \quad 40,7-67,4$ & 17,5 & $7,6-27,4$ \\
\hline \multirow[t]{3}{*}{$2011-12$} & $35-44$ & $49,7 \quad 44,3-55,1$ & $31,5 \quad 26,6-36,7$ & $39,1 \quad 32,8-43,4$ & $27,7 * 23,0-32,8$ & $20,0^{*}$ & $15,7-24,3$ \\
\hline & $45-54$ & $52,7 \quad 46,4-59,2$ & $32,7 \quad 26,9-38,9$ & $43,1 \quad 36,9-48,6$ & $38,2 \quad 32,1-44,5$ & 24,1 & $18,8-29,4$ \\
\hline & $55-65$ & $53,1 \quad 46,1-60,0$ & $39,3 \quad 24,2-37,0$ & $28,9 \quad 22,0-34,5$ & $54,8 \quad 47,8-61,2$ & 35,5 & $29,0-42,0$ \\
\hline
\end{tabular}

${ }^{*}$ p para tendencia en el mismo período $<0,01$.

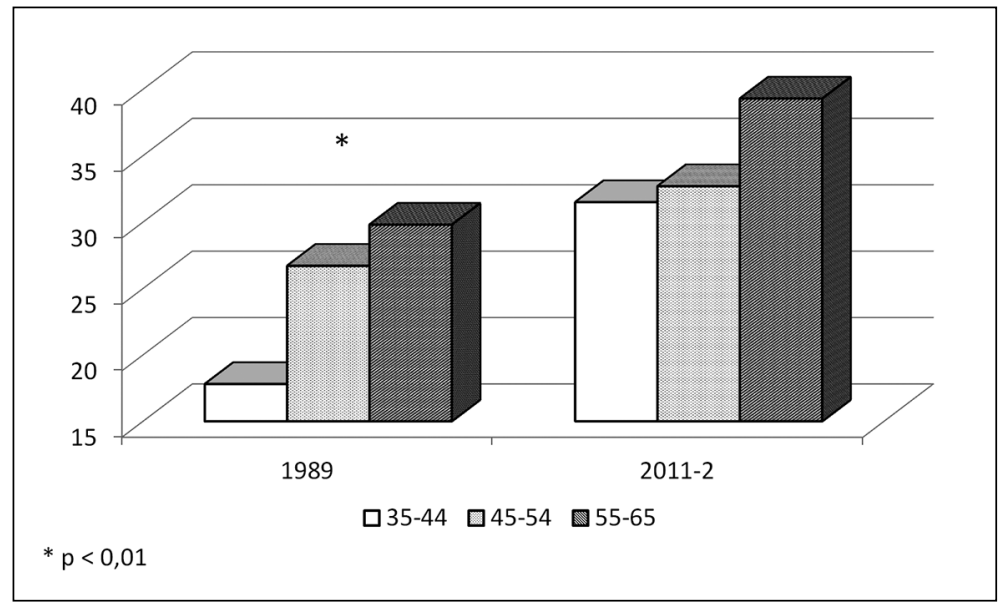

Figura 1. Prevalencia de obesidad según rango de edad. 
Evolución de los factores de riesgo cardiovascular - F. Lanas et al

Tabla 5. Prevalencia de factores de riesgo por nivel educacional e intervalo de confianza de $\mathbf{9 5 \%}$

\begin{tabular}{|c|c|c|c|c|c|c|c|c|c|c|}
\hline \multirow{2}{*}{$\begin{array}{l}\text { Año } \\
1989\end{array}$} & \multirow{2}{*}{$\begin{array}{c}\text { Rango } \\
\text { estudios } \\
\text { (años) } \\
<9\end{array}$} & \multicolumn{2}{|c|}{ Sobrepeso } & \multicolumn{2}{|c|}{ Obesidad } & \multicolumn{2}{|c|}{ Fumador } & $\begin{array}{c}\text { Hipertensión } \\
\text { arterial }\end{array}$ & \multicolumn{2}{|c|}{$\begin{array}{l}\text { Colesterol } \\
\text { elevado }\end{array}$} \\
\hline & & 41,7 & $31,0-52,3$ & 25,0 & $15,7-34,3$ & 19,1 & $10,6-27,5$ & $38,1 * 27,6-48,6$ & 14,3 & $6,8-20,8$ \\
\hline & $9-12$ & 41,9 & $30,6-53,2$ & 27,0 & $16,8-37,2$ & 43,2 & $31,9-54,6$ & $32,4 \quad 21,9-43,2$ & 16,2 & $7,8-24,6$ \\
\hline & $>12$ & 47,6 & $32,3-62,9$ & $16,7^{*}$ & $5,2-28,1$ & 45,2 & $30,0-60,5$ & $21,4 \quad 8,9-34,0$ & 9,5 & $0,6-18,4$ \\
\hline \multirow{3}{*}{$\begin{array}{l}2011- \\
12\end{array}$} & $<9$ & 46,0 & $37,3-54,8$ & 34,1 & $25,8-42,4$ & 38,9 & $30,3-47,4$ & $35,7 \quad 27,3-44,1$ & 23 & $15,7-30,3$ \\
\hline & $9-12$ & 51,5 & $46,4-56,8$ & 33,2 & $28,3-38,2$ & 36,9 & $31,9-41,9$ & $41,1 \quad 36,0-46,3$ & 23,1 & $18,7-27,5$ \\
\hline & $>12$ & 53,9 & $48,4-59,4$ & 29,0 & $23,6-33,5$ & 35,4 & $30,2-40,7$ & $35,5 \quad 30,3-40,9$ & 28,9 & $23,9-33,9$ \\
\hline
\end{tabular}

*p para tendencia en el mismo período $=0,038$.

no influyó en la prevalencia de tabaquismo en ninguno de los 2 períodos. La distribución por edad sin embargo fue diferente en ambos períodos: en 1989 entre los 35-44 años fumaba el 49,4\%, entre los 45-54 el 26,7\% y entre 55-65 años bajó a $17,5 \%$, para los años 2011-12 las proporciones de fumadores fueron $39,1,43,1$ y $28,9 \%$ respectivamente. La edad de comienzo ( 18 y 17,6 años) y el número de cigarrillos por día ( 8,2 y 7,8 cigarrillos/ día) fue similar.

No hubo diferencias significativas en las cifras de presión sistólica y diastólica entre ambos períodos, aunque fue 2 y $1 \mathrm{mmHg}$ menor en 2011-12. La prevalencia de hipertensión arterial aumentó en forma no significativa entre 1989 y 2011-12 de $32,7 \%$ a $38,1 \%$, pero mejoró el grado de conocimiento de ser hipertensión de $33,8 \%$ a $53,9 \%$, el porcentaje en tratamiento de $16,7 \%$ a $32,9 \%$ y el logro de presiones bajo la meta de 6,7 a $13,8 \%$. La prevalencia de hipertensión arterial fue similar entre 1989 y $2011-12$ en el rango de edad de 55-65 años: $54,4 \%$ y $54,8 \%$ respectivamente, pero fue más alta en el segundo período para los sujetos en edades entre $35-44$ años: $19,3 \%$ y $27,7 \%$ y entre $45-54$ años $30 \%$ y $38,2 \%$. No hubo relación entre nivel de educación y prevalencia de hipertensión.

Los niveles de colesterol total promedio subieron $9 \mathrm{mg} / \mathrm{dL}$ en 2011-12 en relación a 1989, y el colesterol no HDL aumentó en 7,4 mg/dL, a pesar que 37 personas usaban estatinas y 4 fibratos en el segundo período y no fueron usados en el primero. La prevalencia de sujetos con colesterol total $\geq 240$ o recibiendo fármacos hipolipidemiantes aumentó significativamente en el segundo período y la prevalencia de sujetos con colesterol total $\geq 200$ $\mathrm{mg} / \mathrm{dL}$ aumentó de $38,5 \%$ a $51,4 \%(\mathrm{p}<0,01)$.
Los niveles de colesterol HDL fueron similaresEn ambos períodos los individuos con peso normal tuvieron niveles promedios de colesterol total más bajos: $179,8 \pm 40,8$ y $192,8 \pm 47,4 \mathrm{mg} / \mathrm{dL}$ en 1989 y 2011-12 respectivamente. El uso de estatinas aumentó por cada grupo etario en el segundo período: $1,2 \%, 5,2 \%$ y 9,5\% aunque no varió según nivel de educación.

No hubo diferencias en la estimación de riesgo de muerte de causa coronaria o IAM a 10 años entre ambos períodos, siendo de $9 \%$ en ambos casos. El riesgo aumentó significativamente en cada tramo de edad, pero no varió por nivel educacional.

\section{Discusión}

Nuestros resultados muestran que en un período de 22 años en hombres entre 35-65 años en población urbana de Temuco hubo un aumento significativoen los años de educación, en la prevalencia de obesidad y colesterol total elevado y de los niveles promedios de colesterol total y colesterol no HDL; hubo una tendencia a aumento de la prevalencia de sobrepeso e hipertensión arterial. No hubo cambios en la prevalencia de tabaquismo, en los niveles de presión arterial y de colesterol HDL. Aumentó la cantidad de personas que conocían su condición de hipertensión y recibían tratamiento para hipertensión o dislipidemia. A pesar de estos cambios el riesgo estimado a 10 años de muerte coronaria e IAM del miocardio no se modificó.

Dentro de las fortalezas de este estudio debe considerarse la similitud en la técnica de muestreo y de determinaciones de presión arterial 
y de niveles de lípidos. Además la muestra es representativa en edad de la población regional, con proporciones para la población por rango de edad de $44,5,31,4$ y $24,1 \%$ y para la muestra de $42,3,30,3$ y $26,8 \%{ }^{23}$. Dentro de las limitaciones de nuestro estudio hay que señalar las que derivan del primer corte transversal: a) la inclusión solamente de hombres, consecuencia de la percepción de esa época que la enfermedad coronaria afectaba fundamentalmente a varones; b) el tamaño muestral que limita el análisis de subgrupos; c) el que no se investigara la presencia de diabetes y no se realizaran determinaciones de glicemia o triglicéridos, esto no permite evaluar cambios en prevalencia de diabetes o intolerancia a glucosa en ayunas ni calcular los niveles de colesterol LDL, pero no afecta el cálculo de riesgo usando las tablas de Framingham ${ }^{20}$. Se ha reportado que el uso de la ecuación de Framingham en poblaciones diferentes a la usada originalmente puede sobreestimar el riesgo ${ }^{24}$, sin embargo, en ausencia de datos validados en una cohorte para Chile se prefirió esta escala por ser la más ampliamente conocida y usada.

El cambio en la prevalencia de factores de riesgo se ha estudiado también en otros países. El British Regional Heart Study estudió el cambio en factores de riesgo cardiovascular en 25 años a partir de 1978 en 7.735 hombres. Hubo reducción de la prevalencia de tabaquismo, presión sistólica y colesterol no HDL, mientras el colesterol HDL, IMC y la actividad física aumentaron. El riesgo de IAM ajustado por edad disminuyó en $62 \%$, siendo la disminución del tabaquismo el principal determinante ${ }^{25}$. En Escocia, entre 1995 y 2009 se observó un incremento de la obesidad, sin cambios en la prevalencia de hipertensión arterial y con disminución de la hipercolesterolemia de 79,6 a 63,8\% ${ }^{26}$. En Finlandia, entre 1992 y 2002, se observó un aumento de circunferencia abdominal y obesidad central en mujeres, pero no en hombres, aumento de intolerancia a glucosa en ayunas y diabetes en ambos sexos, con disminución de presión arterial media y de la prevalencia de hipertensión arterial ${ }^{27}$.

El primer estudio nacional que comparó los cambios de factores de riesgo en una misma población fue realizado en Santiago, donde fueron evaluados en 2 ocasiones: 1988 y 1992. La obesidad aumentó de 6 a $11 \%$ en hombres y de 14 a $24 \%$ en mujeres, aunque hay que considerar el aumento de edad de 5 años de los participantes entre ambas determinaciones ${ }^{10,11}$. La ENS 2009-10 reportó $26,2 \%$ de obesidad en hombres entre 45 64 años ${ }^{14}$ y 2 años después nuestros datos muestran un aumento a $31,5 \%$. Uno de los hallazgos más interesantes de nuestro estudio es el cambio del patrón de obesidad a lo largo del tiempo, en 1989 la obesidad, incluyendo obesidad mórbida, entre 35-44 años era 17,8\% y aumentaba paulatinamente en cada década. En 2011-12 para ese rango de edad la obesidad fue $31,5 \%$, similar al promedio para todo el grupo, y su prevalencia no aumentó en las décadas siguientes. Del mismo modo la obesidad mórbida era sólo $2,4 \%$ entre 35-44 años en 1989 aumentando a 3,5\% entre 55-65 años, en cambio en el período más reciente la más alta prevalencia de obesidad mórbida se encuentra en la década de los más jóvenes, 7,4\%. Dada la relación directa conocida entre obesidad e hipertensión arteria ${ }^{15}$ y colesterol total elevado parece ser esta la razón fundamental para las mayores prevalencias de hipertensión observadas en el último período para los rangos de edad 35-44 y 45-54 años.

No se ha observado una reducción de la proporción de fumadores, aunque hubo un cambio en la proporción de fumadores según nivel de educación, en 1989 los grupos con educación intermedia y alta concentraban la mayor parte de fumadores, en cambio en el período más reciente el grupo con mayor educación fuma algo menos, reflejando posiblemente el impacto de las campañas de educación.

Fasce, determinó los cambios en prevalencia de hipertensión arterial en 1988 y 2004 en población urbana de la VIII Región, describiendo un aumento de prevalencia desde $18,6 \%$ a $21,7 \%$, este aumento se observó en ambos sexos y en todo el rango de edades ${ }^{12}$. Entre la Primera y Segunda ENS, años 2003 y 2010, hubo una disminución en la prevalencia en hombres: 36,7 y $28,7 \%{ }^{13-14}$. Nuestros resultados son concordantes con las observaciones de Fasce, con un aumento de la prevalencia de hipertensión, y esta está determinada por una mayor tasa de hipertensión en los jóvenes. Al comparar ambos períodos se observa una mejoría en el conocimiento de la condición de hipertenso, del porcentaje de sujetos en tratamiento y del logro de metas, sin embargo la magnitud de la mejoría resulta insuficiente para un período de 22 años de políticas públicas orientadas 
a mejorar la cobertura y control de la hipertensión arterial. Como comparación la encuesta de salud de Estados Unidos de Norteamérica, NHANES en el período entre 1988 y 2008 demostró una mejoría del control de la hipertensión arterial de $27,3 \%$ a $51,1 \%{ }^{28}$. Aunque en nuestros estudios no se determinó el conocimiento de tener niveles de colesterol elevados, el uso activo de estatinas por $1,2 \%$ entre 35-44 años, llegando a 9,5\% entre 5565 años demuestra un escaso control poblacional de esta condición.

En los países desarrollados se ha observado una marcada reducción del riesgo cardiovascular en las últimas décadas ${ }^{29}$. En contraste, en nuestro país hubo una escasa reducción de las tasas de mortalidad cardiovascular observada entre 1990 y 2007 , de 161,34 a 156,31 por $100.000^{30}$ lo que es coincidente con nuestra observación de ausencia de cambio en el riesgo cardiovascular, aunque en la mortalidad influyen otros factores como el envejecimiento de la población en Chile y la reducción de letalidad por mejor tratamiento, lo que se ha documentado para el caso del infarto del miocardio $^{31}$. La paradoja de aumento de prevalencia de factores de riesgo sin aumento del riesgo estimado de eventos cardiovasculares se explica porque el riesgo cardiovascular está determinado en un muy alto porcentaje por los niveles de colesterol, presión arterial y tabaquismo en la población. En nuestros datos hay un aumento de prevalencia de hipertensión arterial que se compensa con un mayor porcentaje de sujetos en tratamiento y una leve reducción de las presiones arteriales promedio de la población, la prevalencia de colesterol total y no HDL elevados también está aumentada pero hay un mayor número de individuos en tratamiento con fármacos como estatinas y fibratos con una pequeña diferencia en los niveles de colesterol, y finalmente hubo ausencia de cambios significativos en el consumo de tabaco. Puede estimarse que el mayor esfuerzo en prevención cardiovascular en Chile logró contener el aumento de riesgo esperado por el incremento en la prevalencia de los factores de riesgo, pero que el grado de control de los factores de riesgo no fue suficiente para reducir el riesgo cardiovascular de la población, a diferencia de lo observado en países desarrollados, en que han bajado las tasas de tabaquismo y el grado de control de dislipidemia e hipertensión son más altos, con reducción de la mortalidad cardiovascular a menos de la mitad.

\section{Referencias}

1. Bases de defunciones, disponible en http:www.deis.cl. [Consultado el 05 de abril de 2014].

2. Departamento de Epidemiología. Estudio carga de enfermedad y carga atribuible 2007. 2008; disponible en: http://epi.minsal.cl/epi/html/invest/cargaenf2008/ minuta21-07-2008.pdf [Consultado el 05 de abril de 2014].

3. Ministerio de Salud de Chile. Los Objetivos Sanitarios para la Década 2000-2010 Evaluación de final del período, Grado de cumplimiento de los objetivos de Impacto. 2010, disponible en: http://epi.minsal.cl/epi/html/ sdesalud/OS/EvaluacionObjetivosSanitarios2000-2010. pdf [Consultado el 05 de abril de 2014].

4. Yusuf S, Hawken S, Ounpuu S, Dans T, Avezum A, Lanas F, et al; INTERHEART Study Investigators. Effect of potentially modifiable risk factors associated with myocardial infarction in 52 countries (the INTERHEART study): case-control study. Lancet 2004; 364 (9438): 937-52.

5. Lanas F, Avezum A, Bautista LE, Díaz R, Luna M, Islam S, et al. INTERHEART Investigators in Latin America. Risk factors for acute myocardial infarction in Latin America: the INTERHEART Latin American study. Circulation 2007; 115 (9): 1067-74.

6. O'Donnell MJ, Xavier D, Liu L, Zhang H, Chin SL, RaoMelacini P, et al. INTERSTROKE investigators. Risk factors for ischaemic and intracerebralhaemorrhagic stroke in 22 countries (the INTERSTROKE study): a case-control study. Lancet 2010; 376 (9735): 112-23.

7. Law MR, Morris JK, Wald NJ. Use of blood pressure lowering drugs in the prevention of cardiovascular disease: meta-analysis of 147 randomised trials in the context of expectations from prospective epidemiological studies. BMJ 2009; 338: b1665.

8. Cholesterol Treatment Trialists' Ctt Collaborators. The effects of lowering LDL cholesterol with statin therapy in people at low risk of vascular disease: meta-analysis of individual data from 27 randomised trials. Lancet 2012.

9. Los Objetivos Sanitarios para la Década 2000-2010 Evaluación de final del período, Grado de cumplimiento de los objetivos de Impacto. http://www.redsalud.gov.cl/ portal/url/item/94c89f56c4e270b0e04001011e011c9c.pd. [Consultado el 05 de abril de 2014].

10. Berríos X, Jadue L, Zenteno J, Ross MI, Rodríguez H. Prevalence of risk factors for chronic diseases: a population study in the Metropolitan Area of Santiago, Chile. 1986-1987. Rev Med Chile 1990; 118: 597-604.

11. Berríos X. Risk factors in adult chronic diseases. An example of epidemiologic research. Boletín Esc Medi- 
cina Pontificia Universidad Católica de Chile 1994; 23: 73-89. 23,24.

12. Fasce E, Campos I, Ibáñez P, Flores $M$, Zárate H, Román $\mathrm{O}$, et al. Trends in prevalence, awareness, treatment and control of hypertension in urban communities in Chile. J Hypertens 2007; 25 (9): 1807-11.

13. Encuesta Nacional de salud 2003 www.minsal.cl. [Consultado el 05 de abril de 2014].

14. Encuesta nacional de salud 2009-10, www.minsal.cl. [Consultado el 05 de abril de 2014].

15. Klein S, Burke LE, Bray GA, Blair S, Allison DB, PiSunyer X, et al. Clinical implications of obesity with specific focus on cardiovascular disease: a statement for professionals from the American Heart Association Council on Nutrition, Physical Activity, and Metabolism: endorsed by the American College of Cardiology Foundation. Circulation 2004; 110 (18): 2952-67.

16. Todd Miller M, Lavie CJ, White CJ. Impact of obesity on the pathogenesis and prognosis of coronary heart disease. J Cardio Metab Syndr 2008 Summer; 3(3): 162-7.

17. Ley 19.966: establece un régimen de garantías en salud, (2005). http://www.leychile.cl/Navegar?idNorma= 229834. [Consultado el 05 de abril de 2014].

18. Lanas F, Davis MR, Standen D, Illesca M, Doghetti J, Stockins B. Prevalencia de factores de riesgo cardiovascular en la ciudad de Temuco. Rev Med Chile 1991; 119 (3): 247-51.

19. Rubinstein AL, Irazola VE, Poggio R, Bazzano L, Calandrelli M, Lanas Zanetti FT, et al. Detection and followup of cardiovascular disease and risk factors in the Southern Cone of Latin America: the CESCAS I study. BMJ Open 2011; 1 (1): e000126.

20. Evaluation, and Treatment of High Blood Cholesterol in Adults (Adult Treatment Panel III). Third Report of the National Cholesterol Education Program (NCEP) Expert Panel on Detection, Evaluation, and Treatment of High Blood Cholesterol in Adults (Adult Treatment Panel III) final report. Circulation 2002; 106: 3143-421.

21. Chobanian AV, Bakris GL, Black HR, Cushman WC, Green LA, Izzo JL, et al, and the National High Blood Pressure Education Program Coordinating Committee. The seventh report of the Joint National Committee on prevention, detection, evaluation, and treatment of high blood pressure. The JNC 7 Report. JAMA 2003; 289: 2560-72.

22. Kelsey, et al. Methods in Observational Epidemiology 2nd Edition, Table 12-15.
23. Instituto Nacional de Estadísticas, censo 2002, disponible en http://www.inearaucania.cl/contenido.aspx?id_ contenido $=13$. [Consultado el 05 de abril de 2014].

24. D’Agostino RB, Grundy S, Sullivan LM, Wilson P. Validation of the Framingham coronary heart disease prediction results of a multiple ethnic groups investigation. JAMA 2001; 286: 180-7.

25. Hardoon SL, Whincup PH, Lennon LT, Wannamethee SG, Capewell S, Morris RW. How much of the recent decline in the incidence of myocardial infarction in British men can be explained by changes in cardiovascular risk factors? Evidence from a prospective populationbased study. Circulation 2008; 117 (5): 598-604.

26. Hotchkiss JW, Davies C, Gray L, Bromley C, Capewell $\mathrm{S}$, Leyland $\mathrm{AH}$. Trends in adult cardiovascular disease risk factors and their socio-economic patterning in the Scottish population 1995-2008: cross-sectional surveys. BMJ Open 2011; 1 (1): e000176. Epub 2011 Aug 9. PubMed PMID: 22021783; PubMed Central PMCID: PMC3191578.

27. Hu G, Lindström J, Jousilahti P, Peltonen M, Sjöberg L, Kaaja R, et al. The increasing prevalence of metabolic syndrome among Finnish men and women over a decade. J Clin Endocrinol Metab 2008; 93 (3): 832-6. Epub 2007 Dec 11.

28. Egan BM, Zhao Y, Axon RNUS trends in prevalence, awareness, treatment, and control of hypertension, 1988-2008. JAMA 2010; 303 (20): 2043-50.

29. Go AS, Mozaffarian D, Roger VL, Benjamin EJ, Berry JD, Blaha MJ, et al American Heart Association Statistics Committee and Stroke Statistics Subcommittee. Executive summary: heart disease and stroke estatistics-2014 update: a report from the American Heart Association. Circulation 2014; 129 (3): 399-410.

30. Evolución de la Mortalidad en Chile según causas de muerte y edad 1990-2007. Disponible en http://www. ine.cl/canales/menu/publicaciones/calendario_de_publicaciones/pdf/010211/evo90_07_010211.pdf en región y 42,3 [Consultado el 05 de abril de 2014].

31. Nazzal NC, Campos TP, Corbalán HR, Lanas ZF, Bartolucci JJ, Sanhueza CP, et al; Grupo GEMI; Departamento de Estudios Multicéntricos, Sociedad Chilena de Cardiología y Cirugía Cardiovascular. The impact of Chilean health reform in the management and mortality of ST elevation myocardial infarction (STEMI) in Chilean hospital]. Rev Med Chile 2008; 136 (10): 1231-9. 